\title{
Low systolic blood pressure and self perceived wellbeing in middle aged men
}

\author{
Annika Rosengren, Gösta Tibblin, Lars Wilhelmsen
}

\author{
Abstract \\ Objective-To examine the relation between \\ systolic blood pressure and self perceived wellbeing \\ in 50 year old men.
}

Design-Cross sectional population study of data derived from questionnaires and physical examination as a part of a cardiovascular risk factor survey.

Setting-City of Gothenburg, Sweden.

Study population-776 men from a random population sample of 1016 men aged 50.

Methods-Self perceived wellbeing according to the Gothenburg quality of life questionnaire, which is an assessment of social, physical, and mental wellbeing in 15 different areas.

Main results-Low systolic blood pressure was significantly $(p<0.05)$ related to impaired social wellbeing in four areas: work, home and family, financial situation, and housing. Of the items dealing with physical wellbeing, health, memory, and appetite were significantly related to blood pressure. As regards mental wellbeing, energy and self confidence were significantly related to systolic blood pressure. Diastolic blood pressure was significantly related to housing, memory, energy, patience, and self confidence. In multiple regression analyses that controlled for smoking, stress, physical activity, social activity, and emotional support, poor social, physical, and mental wellbeing were all significantly related to low systolic blood pressure independently of other factors. Low diastolic blood pressure was independently associated with poor physical and mental, but not social, wellbeing.

Conclusion-Low systolic blood pressure was associated with poor perception of wellbeing in several areas. The cause is unclear.

\section{Introduction}

Most epidemiological research deals with well defined disease entities such as coronary heart disease or cancer, or with mortality. Poor health, however, is not confined to severe or life threatening diseases and may not always be attributable to causes that are easily defined. In the study of health in the population, attention should also be directed towards the determinants of less specific forms of impaired health.

Interest was recently focused on the possible ill effects of low, compared with normal or high blood pressure. ${ }^{1-3}$ As opposed to the evident effect of high blood pressure on cardiovascular morbidity and mortality, low blood pressure has been associated with tiredness and feeling faint ${ }^{12}$ as well as with dizziness and minor psychiatric illness. ${ }^{3}$ The reasons for these findings remain obscure, although cerebral hypoperfusion or different degrees of monoaminergic activity have been considered. ${ }^{3}$

In Gothenburg, cohorts of 50 year old men born in 1913, 1923, and 1933 have been examined since 1963 to monitor population changes in cardiovascular morbidity and mortality. The youngest of these cohorts, men born in 1933, was examined in 1983-4. In addition to conventional cardiovascular risk factors the study protocol included several psychosocial factors to determine the potential impact of these factors on various aspects of health. The Gothenburg quality of life instrument ${ }^{4}$ has been used in these cohort studies since 1973. In view of the recent reports on low blood pressure and various psychological symptoms ${ }^{1-3}$ we set out to examine if there might also be a relation between blood pressure and self perceived wellbeing.

Methods

STUDY POPULATION

In 1983 a random sample was drawn of half of all men in Gothenburg who were born in 1933. The men in the sample $(n=1016)$, all of whom were 50 years old, were invited to a health examination; $776(76 \%)$ responded. No men had to be excluded from the study because of somatic or psychiatric disease.

\section{EXAMINATIONS}

All examinations were performed in the morning. One blood pressure reading was taken with the subject sitting, after five minutes' rest, with a Hawkesley random zero mercury sphygmomanometer. Body weight was measured on a lever balance to the nearest $0 \cdot 1 \mathrm{~kg}$. Body mass index was calculated as weight $(\mathrm{kg}) /$ height $\left(\mathrm{m}^{2}\right)$. All blood samples were taken after an overnight fast. Serum concentrations of cholesterol and triglycerides were determined according to standard laboratory procedures.

Before the examination all participants had completed a postal questionnaire dealing with current occupation; smoking habits; physical activity at work and during leisure; presence of diabetes; treatment for hypertension, chest pain, or dyspnoea; and presence of psychological stress. The answers were checked by the examining physician. Leisure time physical activity was coded on a four point scale, with 1 representing sedentary activity, 2 representing moderate exercise like walking or light gardening work during at least four hours per week, and 3-4 representing regular, strenuous, or very strenuous activity. Psychological stress (defined as feeling tense, irritable, filled with anxiety, or having difficulty sleeping as a result of conditions at work or at home 5 was graded from 1 , never experienced stress, to 5 and 6 , a permanent feeling of stress during either the past year or the past five years. Grades 5 and 6 were defined as heavy stress.

A Rose questionnaire on the presence of angina was included. ${ }^{6}$ Subjects admitting to any chest pain were further evaluated by the examining physician, and angina was diagnosed or suspected if the person had chest pain brought on by exertion and relieved within 10 minutes by stopping or slowing down the activity that brought on the pain.

During the examination the participants completed a detailed questionnaire about social variables. Self perceived quality of life was investigated by assessing social, physical, and mental wellbeing in 15 different areas $^{4}$ on a scale with seven ratings for each item, ranging from $1=$ excellent, could not be better to $7=$ very poor. Social wellbeing included the dimensions of work, home and family, financial situation, 
and housing. Physical wellbeing included health, hearing, vision, memory, fitness, and appetite. Mental wellbeing included mood, energy, patience, self confidence, and sleep. The proportion of subjects reporting excellent wellbeing (ratings 1-2) has been shown to be stable over time $e^{4}$ and was in good agreement with the distribution for the subjects of the present study. A highly significant correlation has been shown between hearing as assessed by the wellbeing scale and tone audiometry. ${ }^{4}$ Excellent wellbeing varied for the different variables. The highest proportion of subjects $(70 \%-80 \%)$ reported excellent wellbeing in previously studied populations, as well as in the present study, for family, housing, appetite, and mood. The lowest proportion of subjects $(30 \%-40 \%)$ reported excellent wellbeing for fitness, vision, and memory. On the other eight variables $50 \%-60 \%$ of subjects reported excellent wellbeing.

A condensed version of the interview schedule for social interaction ${ }^{7}$ was used to measure social support. This instrument yields two scales describing the deep emotional relationships or "attachment" on one hand and the more peripheral contacts of social networks and "social integration" on the other. In our study, both scales were dichotomised into low and high, with low defined as the lowest $25 \%$ of the distribution.

The men were also asked how active they had been during the year preceding the examination. ${ }^{8}$ Activities were divided into home activities (repairs, hobbies, studying, reading, gardening, etc), activities outside the home (swimming, cycling, skiing, theatre, sports events, horse racing events, visiting restaurants, travelling, etc) and social activities (parties at home, organised sport, visiting relatives or friends, trade union or other association meetings, etc). The responses never, occasionally, or often/regularly were given a score of 0,1 , and 2 respectively and graded for each of the three activity areas.

TABLE I-Correlations between variables indicating wellbeing and blood pressure

\begin{tabular}{|c|c|c|c|c|c|c|c|c|}
\hline & \multicolumn{4}{|c|}{ Systolic blood pressure } & \multicolumn{4}{|c|}{ Diastolic blood pressure } \\
\hline & \multicolumn{2}{|c|}{$\begin{array}{l}\text { Correlation } \\
\text { coefficient }\end{array}$} & \multicolumn{2}{|c|}{$\mathrm{p}$ Value } & \multicolumn{2}{|c|}{$\begin{array}{l}\text { Correlation } \\
\text { coefficient }\end{array}$} & \multicolumn{2}{|c|}{$\mathrm{p}$ Value } \\
\hline Social variables & & $-0 \cdot 12$ & & 0.001 & & -0.087 & & 0.016 \\
\hline Situation at work & -0.071 & & 0.050 & & -0.059 & & $0 \cdot 10$ & \\
\hline Home and family & -0.074 & & 0.040 & & -0.044 & & $0 \cdot 23$ & \\
\hline Financial situation & $-0 \cdot 10$ & & 0.005 & & -0.070 & & 0.052 & \\
\hline Housing & $-0 \cdot 12$ & & 0.001 & & $-0 \cdot 11$ & & 0.003 & \\
\hline Physical variables & & $-0 \cdot 11$ & & 0.003 & & -0.095 & & 0.008 \\
\hline Health & -0.095 & & 0.008 & & $-0 \cdot 11$ & & 0.002 & \\
\hline Hearing & -0.069 & & 0.055 & & -0.035 & & $0 \cdot 33$ & \\
\hline Vision & -0.055 & & $0 \cdot 13$ & & -0.063 & & 0.08 & \\
\hline Memory & $-0 \cdot 15$ & & 0.0001 & & -0.094 & & 0.010 & \\
\hline Fitness & 0.021 & & 0.57 & & 0.013 & & $0 \cdot 71$ & \\
\hline Appetite & $-0 \cdot 10$ & & 0.005 & & $-0 \cdot 11$ & & 0.002 & \\
\hline Mental variables & & -0.094 & & $0 \cdot 010$ & & -0.096 & & 0.008 \\
\hline Mood & 0.062 & & 0.087 & & -0.068 & & 0.059 & \\
\hline Energy & $0 \cdot 11$ & & 0.003 & & $-0 \cdot 11$ & & 0.004 & \\
\hline Patience & -0.063 & & 0.080 & & $-0 \cdot 11$ & & 0.003 & \\
\hline Self confidence & -0.080 & & 0.026 & & -0.072 & & 0.046 & \\
\hline Sleep & -0.051 & & $0 \cdot 16$ & & -0.027 & & 0.45 & \\
\hline
\end{tabular}

STATISTICAL METHODS

Correlations between systolic and diastolic blood pressure and continuous or graded variables, or both, were tested with Pearson's test. Multiple regression analyses were performed with sums of social, physical, and mental wellbeing as dependent variables and with insignificant variables $(p>0 \cdot 15)$ excluded in a stepwise manner.

\section{Results}

Table I shows the correlations between each of the 15 wellbeing variables and systolic and diastolic blood pressure. All four items describing social wellbeing were related to systolic blood pressure, with low blood pressure associated with poorer self perceived quality of life. However, with the exception of housing, there were no correlations with diastolic blood pressure. Three of the six items dealing with physical wellbeing were associated with blood pressure. Poor perceived memory was strongly associated with low systolic, as well as diastolic, blood pressure. Similar, less strong associations were found for health and appetite. Two of the five items dealing with mental wellbeing, energy and patience, were related to systolic as well as to diastolic blood pressure, whereas self confidence was related to diastolic blood pressure only. The relations between low systolic and diastolic blood pressure and impaired social, physical, and mental wellbeing were all significant.

The men in the study were divided into fifths according to their systolic blood pressure. Table II shows ratings for social, physical, and mental wellbeing for each fifth and lists potential confounders of the inverse association between wellbeing and blood pressure. Both serum cholesterol and serum triglyceride concentrations were positively related to systolic blood pressure and so was body mass index. A slightly higher proportion of the men with low blood pressure were smokers $(p=0 \cdot 06)$. Of the psychosocial variables, home activities and activities outside the home were positively associated with systolic blood pressure, whereas social activities were not, nor were leisure time physical activity, social integration, psychological stress, or occupational class. There was a weak association between low emotional support and low systolic blood pressure.

Cardiovascular and other symptoms, including angina, were not associated with systolic blood pressure (table III). Blood pressure was not significantly related to regular contact with a physician for symptoms or illness. When men who were treated for hypertension were excluded from the analysis there was still no association between contact with a physician and systolic blood pressure (data not shown).

To allow for the potential confounding effects of other factors influencing systolic blood pressure and wellbeing, multiple regression analyses were per-

TABLE II-Social, physical, and mental quality of life, cardiovascular risk factors, and psychosocial factors by fifths of systolic blood pressure

\begin{tabular}{|c|c|c|c|c|c|c|}
\hline \multirow[b]{2}{*}{ Variable } & \multicolumn{5}{|c|}{ Systolic blood pressure } & \multirow[b]{2}{*}{ p Value } \\
\hline & $\leqslant \underset{(n=142)}{118 \mathrm{~mm} H g}$ & $\underset{(\mathrm{n}=184)}{120-128 \mathrm{~mm} \mathrm{Hg}}$ & $\begin{array}{c}130-136 \mathrm{~mm} \mathrm{Hg} \\
(\mathrm{n}=145)\end{array}$ & $\begin{array}{c}138-148 \mathrm{~mm} \\
(\mathrm{n}=157)\end{array}$ & $>\underset{(n=148)}{148 \mathrm{~mm} \mathrm{Hg}}$ & \\
\hline Mean (SD) social quality of life & $10 \cdot 0(4 \cdot 7)$ & $9 \cdot 4(4 \cdot 1)$ & $9 \cdot 6(3 \cdot 7)$ & $8 \cdot 7(4 \cdot 0)$ & $8 \cdot 7(3 \cdot 9)$ & 0.001 \\
\hline Mean (SD) physical quality of life & $17 \cdot 5(6 \cdot 0)$ & $17 \cdot 6(5 \cdot 2)$ & $16 \cdot 7(4 \cdot 7)$ & $16 \cdot 3(4.9)$ & $16 \cdot 3(5 \cdot 1)$ & 0.003 \\
\hline Mean (SD) mental quality of life & $13 \cdot 2(6 \cdot 4)$ & $12 \cdot 2(5 \cdot 0)$ & $12 \cdot 3(4 \cdot 6)$ & $11 \cdot 4(5 \cdot 2)$ & $12 \cdot 0(5 \cdot 5)$ & 0.010 \\
\hline Mean (SD) serum cholesterol $(\mathrm{mmol} / \mathrm{l})$ & $6 \cdot 0(1 \cdot 2)$ & $6 \cdot 1(1 \cdot 1)$ & $6 \cdot 2(1 \cdot 1)$ & $6 \cdot 1(1 \cdot 0)$ & $6 \cdot 2(1 \cdot 2)$ & 0.021 \\
\hline Mean (SD) serum triglyceride (mmol/l) & $1.3(0.8)$ & $1.3(0.8)$ & $1 \cdot 4(1 \cdot 3)$ & $1.4(0.9)$ & $1.8(1.5)$ & 0.0008 \\
\hline Mean (SD) body mass index $\left(\mathrm{kg} / \mathrm{m}^{2}\right)$ & $24.3(3.5)$ & $25 \cdot 3(3 \cdot 2)$ & $25 \cdot 8(3 \cdot 2)$ & $25.9(3.3)$ & $26.9(3.4)$ & 0.0001 \\
\hline$\%$ (No) current smokers & $41(58)$ & $41(75)$ & $34(49)$ & $34(54)$ & $34(51)$ & 0.065 \\
\hline$\%$ (No) low home activity (score 1 ) & $31(44)$ & $27(49)$ & $26(37)$ & $22(35)$ & $22(33)$ & 0.038 \\
\hline$\%$ (No) low activity outside home (score 1 ) & $31(44)$ & $28(52)$ & $25(36)$ & $27(43)$ & $18(26)$ & 0.015 \\
\hline$\%$ (No) low social activity (score 1 ) & $32(45)$ & $32(59)$ & $27(39)$ & $27(43)$ & $29(43)$ & 0.66 \\
\hline$\%$ (No) low leisure time physical activity (score 1 ) & $31(44)$ & $25(46)$ & $13(19)$ & $20(32)$ & $20(30)$ & 0.51 \\
\hline$\%$ (No) low social integration (score 1 ) & $21(30)$ & $21(38)$ & $23(33)$ & $19(30)$ & $20(30)$ & 0.80 \\
\hline$\%$ (No) low emotional support (score 1 ) & $25(35)$ & $22(40)$ & $28(40)$ & $20(32)$ & $18(26)$ & 0.036 \\
\hline$\%$ (No) heavy stress (rating 5-6) & $13(19)$ & $12(21)$ & $14(20)$ & $12(19)$ & $17(25)$ & $0 \cdot 24$ \\
\hline$\%(\mathrm{No})$ manual workers & $38(54)$ & $42(77)$ & $39(57)$ & $44(69)$ & $38(56)$ & 0.67 \\
\hline
\end{tabular}




\begin{tabular}{|c|c|c|c|c|c|c|}
\hline \multirow[b]{2}{*}{ Symptom } & \multicolumn{5}{|c|}{ Systolic blood pressure } & \multirow[b]{2}{*}{$\begin{array}{l}p \text { Value } \\
\text { for trend }\end{array}$} \\
\hline & $\leqslant \underset{(n=142)}{118 \mathrm{~mm} \mathrm{Hg}}$ & $\begin{array}{c}120-128 \mathrm{~mm} \\
(\mathrm{n}=184)\end{array}$ & $\begin{array}{c}130-136 \mathrm{~mm} \mathrm{Hg} \\
(\mathrm{n}=145)\end{array}$ & $\begin{array}{c}138-148 \mathrm{~mm} \\
(\mathrm{n}=157)\end{array}$ & $\begin{array}{c}>148 \mathrm{~mm} \mathrm{Hg} \\
(\mathrm{n}=148)\end{array}$ & \\
\hline \multicolumn{3}{|l|}{ Seen regularly by a physician because of } & $30(43)$ & $27(41)$ & $37(55)$ & 0.088 \\
\hline Pharmacological treatment of hypertension & $2(3)$ & $4(8)$ & $8(12)$ & $13(20)$ & $24(36)$ & 0.001 \\
\hline Known diabetes & $5(7)$ & $1(2)$ & $6(4)$ & $1(2)$ & $7(10)$ & $0 \cdot 38$ \\
\hline Pain in calves when walking & $11(16)$ & $8(15)$ & $11(16)$ & $11(17)$ & $10(15)$ & 0.63 \\
\hline Chest pain walking uphill & $13(19)$ & $9(17)$ & $14(20)$ & $9(14)$ & $8(12)$ & $0 \cdot 29$ \\
\hline Dyspnoea walking two flights of stairs & $23(33)$ & $21(38)$ & $17(24)$ & $21(33)$ & $21(30)$ & 0.66 \\
\hline \multirow{2}{*}{$\begin{array}{l}\text { Angina pectoris (definite or suspected- } \\
\text { physician's diagnosis) }\end{array}$} & & & & & & \\
\hline & $7(10)$ & $3(6)$ & $6(8)$ & $4(7)$ & $7(11)$ & $0 \cdot 77$ \\
\hline
\end{tabular}

TABLE IV-Multiple regression analyses of social, physical, and mental wellbeing

\begin{tabular}{|c|c|c|c|c|c|c|}
\hline \multirow[b]{3}{*}{ Independent variables } & \multicolumn{6}{|c|}{ Dependent variables } \\
\hline & \multicolumn{2}{|c|}{ Social wellbeing } & \multicolumn{2}{|c|}{ Physical wellbeing } & \multicolumn{2}{|c|}{ Mental wellbeing } \\
\hline & Coefficient (SE) & $\mathrm{p}$ Value & Coefficient (SE) & $\mathrm{p}$ Value & Coefficient (SE) & $\mathrm{p}$ Value \\
\hline \multicolumn{7}{|c|}{ Systolic blood pressure } \\
\hline Intercept & $21 \cdot 58(1.43)$ & 0.0001 & $31.95(1.82)$ & 0.0001 & $25.80(1.84)$ & 0.0001 \\
\hline Systolic blood pressure $(\mathrm{mm} \mathrm{Hg})$ & $-0.023(0.008)$ & $0 \cdot 006$ & $-0.026(0.010)$ & 0.015 & $-0.025(0.011)$ & 0.018 \\
\hline Current smoking $($ no $=0$, yes $=1)$ & $0.55(0.29)$ & $0 \cdot 060$ & $0.71(0.38)$ & 0.059 & NA & \\
\hline Heavy psychological stress $($ no $=0$, yes $=1)$ & $1.39(0.41)$ & 0.0006 & $1.27(0.52)$ & 0.014 & $3.02(0.53)$ & 0.0001 \\
\hline Leisure time physical activity (score $1-4$ ) & NA & & $-1.44(0.31)$ & 0.0001 & $-0.99(0.32)$ & 0.0018 \\
\hline Social activities, total (score $0-64$ ) & $-0.093(0.016)$ & 0.0001 & $-0.091(0.022)$ & 0.0001 & $-0.094(0.023)$ & 0.0001 \\
\hline Emotional support $($ low $=0$, high $=1)$ & $-1.91(0.34)$ & 0.0001 & $-1.56(0.43)$ & 0.0003 & $-1.94(0.45)$ & 0.0001 \\
\hline \multicolumn{7}{|c|}{ Diastolic blood pressure } \\
\hline Intercept & $20 \cdot 50(1 \cdot 45)$ & 0.0001 & $31.50(1.84)$ & 0.0001 & $25.63(1.87)$ & 0.0001 \\
\hline Diastolic blood pressure $(\mathrm{mm} \mathrm{Hg})$ & $-0.021(0.013)$ & $0 \cdot 11$ & $-0.034(0.017)$ & $0 \cdot 040$ & $-0.037(0.017)$ & 0.030 \\
\hline Current smoking $($ no $=0$, yes $=1)$ & $0.56(0 \cdot 29)$ & 0.057 & $0.71(0.38)$ & 0.063 & NA & \\
\hline Heavy psychological stress $($ no $=0$, yes $=1)$ & $1.35(0.41)$ & 0.0009 & $1.23(0.52)$ & 0.017 & $2.98(0.53)$ & 0.0001 \\
\hline Leisure time physical activity (score $1-4$ ) & NA & & $-1 \cdot 44(0.31)$ & 0.0001 & $-0.99(0.32)$ & 0.0019 \\
\hline Social activities, total (score $0-64$ ) & $-0.094(0.016)$ & 0.0001 & $-0.092(0.022)$ & 0.0001 & $-0.094(0.023)$ & 0.0001 \\
\hline Emotional support $($ low $=0$, high $=1)$ & $-1.94(0.34)$ & 0.0001 & $-1.57(0.44)$ & 0.0003 & $-1.94(0.45)$ & 0.0001 \\
\hline
\end{tabular}

NA= omitted in the final version because of non-significance $(p>0 \cdot 15)$.

formed with the composite measures of social, physical and mental wellbeing as dependent variables (table IV). Blood pressure was entered as a continuous variable, and the other variables were entered as indicated in table IV. Total social activities denote a summary score of all three activity scores. Physical wellbeing was approximately normally distributed, whereas the sums of social and mental wellbeing were skewed towards lower scores. As the population was comparatively large and log transforming the data changed very little in the analyses we used the untransformed data. Of the variables significantly associated with systolic blood pressure, serum cholesterol, body mass index, and drug treatment for hypertension were not significantly associated with any of the three dimensions of wellbeing in any of the regression analyses, so these variables were not included in the final analyses. Impaired social, physical, and mental wellbeing were all significantly related to low. systolic blood pressure independent of smoking; heavy psychological stress; leisure time physical activity; social activities; and emotional support. In similar multiple regression analyses low diastolic blood pressure was associated with impaired physical and mental wellbeing but not with social wellbeing.

\section{Discussion}

In Scandinavia as well as in Anglo-Saxon countries low blood pressure is not regarded as a disease by the medical profession, and in textbooks of medicine low blood pressure, in the absence of other underlying conditions, is treated summarily or not at all. Nevertheless there is a widespread popular belief that low blood pressure signals fatigue and lack of energy. The findings of the present study may reflect people with low blood pressure having altered their perception of their quality of life after having been informed about their low blood pressure. We could not examine this in the present study, but it is unlikely to have influenced our results to any great extent as in clinical settings people with low blood pressure are generally reassured because of their lower risk for cardiovascular complications.

The past few years have seen the publication of a few studies that have tended to support the popular belief that low blood pressure is associated with various symptoms. ${ }^{1-39}$ In 1987 Pemberly used Australian data to analyse symptoms of palpitation of the heart, dizziness, fainting, and tiredness in relation to blood pressure. ${ }^{2}$ In women, but not in men, a significant association was found between low blood pressure and tiredness, but not dizziness. The British health and lifestyle survey found associations between low systolic blood pressure and both tiredness and feelings of faintness.' ${ }^{1}$ These associations were independent of several potentially confounding factors. Another British study found dizziness or giddiness in men and unexplained tiredness in women to be related to low systolic blood pressure. ${ }^{3}$ Minor psychological symptoms, as assessed with the general health questionnaire, ${ }^{10}$ were also associated with low systolic blood pressure, and the association between low blood pressure and physical symptoms was no longer significant when the general health questionnaire score was controlled for. It was concluded that the physical symptoms of dizziness or giddiness and tiredness probably were secondary to the primary disturbance in mental state.

Our study did not include measurements of psychological disturbances such as worry, restlessness, strain, feelings of depression, and lack of hope or other components of the general health questionnaire. Some of these items are reflected in our single question on psychological stress, which in the questionnaire was defined as feeling tense, irritable, filled with anxiety, or having sleeping difficulties as a result of conditions at work or at home. This variable has not been validated against other measures of mental health but has been shown to be an independent predictor of mortality and of coronary heart disease. ${ }^{5}$ Controlling for psychological stress did not alter the association between the 
wellbeing scales and blood pressure. The subscale for mental wellbeing might also be used as an indicator of mental health. All three subscales were strongly intercorrelated, but if mental wellbeing was entered as an independent variable in the multiple regression analyses concerning the two other subscales, blood pressure no longer made an independent contribution. This could possibly point to a primary mental disturbance in the association between low blood pressure and self perceived quality of life, but the strong association between mental wellbeing and the two other subscales precludes firm conclusions.

In the cohort of men born in 1913 and examined in 1963 , low blood pressure was associated with a history of pulmonary tuberculosis or hospitalisation for pneumonia. ${ }^{11}$ No similar data were collected for the present cohort, but official statistics show a drastic decrease of these diseases throughout this century, and they are very unlikely to have mattered in the present study.

The Michigan statewide blood pressure survey found an association between high blood pressure and low self confidence in combination with low perceived control but also with poorer self reported mental health. ${ }^{12}$ Our study did not find any psychosocial factor, including low socioeconomic status and poor social network, to be associated with high blood pressure. On the contrary, low perception of wellbeing, poor emotional support, and low activity were all associated with low blood pressure. The difference between the results of the present study and the Michigan study ${ }^{12}$ is hard to explain, but may be due to different methods of assessing these psychosocial constructs, and also that the Michigan study used subjects from a much wider age span. Self confidence, measured according to the Gothenburg instrument, has been shown to deteriorate significantly with age. ${ }^{13}$

Other studies dealing with the associations between low blood pressure and symptoms have excluded people being treated for hypertension from the analyses. ${ }^{3}$ We did not exclude men who had drug treatment for hypertension as this would have meant subtracting a large number of men with potentially poorer quality of life from the highest blood pressure fifth. However, a comparison of those being treated for hypertension with the rest of the men with regard to self perceived wellbeing yielded significant differences only for the variables health ( 3.1 in hypertensive men $v$ 2.7 in the rest; $p=0.033)$ and physical fitness $(4.2 v 3.6$; $\mathrm{p}=0.0008$ ). Excluding all men treated for hypertension from the analyses of the relations between systolic blood pressure and self perceived wellbeing made little difference to the results.

Self perceived wellbeing is, by definition, subjective. An excellent correlation has been shown in the study of men born in 1913 and 1923 between the quality of life item hearing and tone audiometry, as well as with the item family and marital state. ${ }^{13}$ However, the Gothen- burg quality of life instrument has not been validated against other quality of life measurements, which is a limitation of the present study. Studies using better validated measurements of quality of life are necessary before a link between low blood pressure and quality of life can be definitely established.

Only a single reading of blood pressure was taken, which would tend to reduce the precision of the blood pressure variable. ${ }^{14}$ The differences in perceived wellbeing were not large but-except for vision, physical capacity, and sleep-they were very consistent. Given the imprecision inherent both in the quality of life instrument and in the single blood pressure reading, the true association between low blood pressure and poor wellbeing may be greater than that indicated in the present study.

In a cross sectional analysis it is difficult to make assumptions on causality. The image evoked by the data may be one of an inactive person lacking in vitality and enjoyment of life, or perhaps of the classic neurasthenic person. However, it is evident that a syndrome of hypotension, if it exists, is rather more than only tiredness and giddiness. Our findings are compatible with the minor psychiatric dysfunction proposed by Pilgrim. ${ }^{3}$

This study was supported by the Swedish Heart and Lung Foundation, the Swedish Labour Market Insurance Company, the Gothenburg Medical Society, and the Knut and Alice Wallenberg Foundation.

1 Wessely S, Nickson J, Cox B. Symptoms of low blood pressure: a population study. $B M Y$ I 1990;301:362-5.

2 Pemberton J. Does constitutional hypotension exist? BMF 1989;298:660-2.

3 Pilgrim JA, Stansfeld S, Marmot M. Low blood pressure, low mood? $B M \mathcal{J}$ 1992;304:75-8.

4 Tibblin G, Tibblin B, Peciva S, Kullman S, Svärdsudd K. The Göteborg quality of life instrument. An assessment of well-being and symptom among men born 1913 and 1923. Methods and validity. Scand $\mathcal{F}$ Prim Healt Care 1990;(suppl 1):33-8.

5 Rosengren A, Tibblin G, Wilhelmsen L. Self-perceived psychological stres and incidence of coronary artery disease in middle-aged men. $\mathrm{Am} \mathcal{F}$ Cardio 1991;68:1171-5.

6 Rose GA, Blackburn H, Gillum RF, Prineas RJ. Cardiovascular survey methods. 2nd ed. Geneva: World Health Organisation, 1982.

7 Undén A-L, Orth-Gomér K. Development of a social support instrument for use in population surveys. Soc Sci Med 1989;29:1387-92.

8 Welin L, Tibblin G, Svärdsudd K, Tibblin B, Ander-Peciva S, Larsson B. Prospective study of social influences on mortality. The study of men born in 1913 and 1923. Lancet 1985;i:915-8.

9 Bengtsson C, Edström K, Furunes B, Sigurdsson JA, Tibblin G. Prevalence of subjectively experienced symptoms in a population sample of women with special reference to women with arterial hypertension. Scand f Prim Health special reference to

10 Goodchild M, Duncan-Jones P. Chronicity and the general health questionnaire. Br F Psychiatry 1985;146:55-61.

11 Tibblin G. High blood pressure in men aged 50. A population study of men born in 1913 [dissertation]. Lenbu: University of Gothenburg, 1967.

12 Cottington EM, Brock BM, House JS, Hawthorne VM. Psychosocial factor and blood pressure in the Michigan statewide blood pressure survey. Am $\mathcal{F}$ Epidemiol 1985;121:515-29.

13 Tibblin G, Cato K, Svärdsudd K. Göteborg quality of life study of men born in 1913 and 1923-age, sex, job satisfaction and cardiovascular diseases. Scand f Prim Health Care 1990;(suppl 1):39-45.

14 MacMahon S, Peto R, Cutler J, Collins R, Sorlie P, Neaton J, et al. Blood pressure, stroke and coronary heart disease. Part 1 , prolonged differences in blood pressure: Prospective observational studies corrected for the regression dilution bias. Lancet 1990;335:765-74.

(Accepted 10 November 1992) 